\title{
Inferencias paleoclimáticas para el Mioceno tardío en la cuenca de Angastaco basadas en el análisis fisionómico foliar: Formación Palo Pintado, Salta, Argentina
}

\author{
*Juan M. Robledo", ${ }^{1,}$, Maricel Y. Horn', Claudia I. Galli ${ }^{3,4}$, Luisa M. Anzótegui ${ }^{1}$ \\ Centro de Ecología Aplicada del Litoral (CECOAL), CONICET, Ruta 5, km 2,5, Casilla correo 291, 3400, Corrientes, Argentina. \\ manuelrobledo@conicet.gov.ar; yaninamaricelh@gmail.com; luisaanzotegui@yahoo.com.ar. \\ 2 Facultad de Ciencias Exactas y Naturales y Agrimensura, Universidad Nacional del Nordeste (FaCENA-UNNE), Av. Libertad 5470, \\ 3400, Corrientes, Argentina. \\ 3 INECOA-Facultad de Ingeniería, Universidad Nacional de Jujuy, Facultad de Ciencias Naturales, Universidad Nacional de Salta, \\ Argentina. \\ 4 INECOA-CONICET, Instituto de Geología y Minería, S.S. de Jujuy, Universidad Nacional de Salta, Argentina. \\ cgalli1801@gmail.com. \\ *Autor de correspondencia: manuelrobledo@conicet.gov.ar
}

\begin{abstract}
RESUMEN. Las rocas sedimentarias continentales que constituyen la Formación Palo Pintado, de edad del Mioceno tardío, afloran en la provincia de Salta y suscitan un gran interés paleoclimático, dadas las condiciones ambientales reinantes durante ese intervalo geocronológico. Los registros geológicos y paleobotánicos sugieren que durante la acumulación de las rocas sedimentarias de la Formación Palo Pintado (cuenca de Angastaco) habrían existido condiciones más húmedas en comparación a las de otras formaciones cercanas y coetáneas, por ejemplo, la Aloformación Playa del Zorro y la Formación Chiquimil (Mioceno tardío, provincias de Catamarca y Tucumán) y las formaciones Salicas y Toro Negro (Mioceno tardío, provincia de La Rioja). En este estudio se analizaron el margen y el área foliar de las hojas fósiles contenidas en las rocas de la Formación Palo Pintado, a fin de obtener la temperatura media anual (TMA) y la precipitación media anual (PMA). Los valores resultantes fueron los siguientes: TMA $=23,98{ }^{\circ} \mathrm{C}$ y $P M A=330,8 \mathrm{~mm}$. Estos resultados se condicen con la interpretación de diferentes autores quienes consideran que la Formación Palo Pintado se habría depositado bajo un ambiente relativamente húmedo, posiblemente como consecuencia de las lluvias caídas localmente en la región de la cuenca de Angastaco.
\end{abstract}

Palabras clave: Neógeno, Paleoclima, Noroeste de Argentina, Mioceno tardio, Salta.

\begin{abstract}
Paleoclimatic inferences for the late Miocene of the Angastaco Basin based on the foliar physiognomic analysis: Palo Pintado Formation, Salta, Argentina. The continental sedimentary rocks that constitute the Palo Pintado Formation of the late Miocene from Salta province, presents a great paleoclimatic interest due to the environmental conditions prevailing during this geochronologic interval. The geological and paleobotanical data suggest that during the sedimentary rocks accumulation of the Palo Pintado Formation (Angastaco Basin), wetter conditions would have existed comparing with other nearby and contemporary Formations, for example the Playa del Zorro Aloformation (late Miocene of Catamarca) and the Chiquimil (late Miocene of Tucumán), Salicas and the Toro Negro Formations (both from the late Miocene of La Rioja). In this study, the margin and the foliar area of the leaves contained on rocks from the Palo Pintado Formation are analyzed, in order to obtain the mean annual temperature (MAT) and the mean annual precipitation (MAP). The resulting values were: $23.98^{\circ} \mathrm{C}$ and $330.8 \mathrm{~mm}$. These results are coincident by the interpretation of different authors, who consider that the Palo Pintado Formation would have been deposited under a relatively humid environment, possibly as a consequence of the rains that affected locally the Angastaco basin region
\end{abstract}




\section{Introducción}

Las rocas sedimentarias de la Formación Palo Pintado (Salta, Argentina) contienen una importante diversidad de fósiles, entre los que predominan las improntas foliares y los palinomorfos (Anzótegui et al., 2017). La inferencia paleoclimática, realizada a través del análisis de estos fitofósiles y de la sedimentología (Herbst et al., 1987; Acevedo et al., 1997; Starck y Anzótegui, 2001; Anzótegui, 2006; Galli et al., 2011), señala que la depositación de las rocas de esta formación se habría desarrollado bajo un clima cálido-húmedo, semejante a las condiciones halladas actualmente en las estribaciones andinas orientales y en el "Chaco Salteño". En esta formación se describen las siguientes paleocomunidades vegetales: dulciacuícola, palustre y bosque higrófilo (Galli et al., 2011). Durante el Mioceno tardío, estas condiciones ambientales resultan atípicas para el ambiente predominante en la región y a escala global (Zachos et al., 2001; Herbert et al., 2016). Para esta época, se infiere un ambiente preferentemente seco que persiste desde el Mioceno temprano (Garralla et al., 2016), el cual queda evidenciado en los registros paleobotánicos de la Formación Chiquimil y la Aloformación Playa del Zorro (Anzótegui, 2004; Garralla et al., 2012), ambas coetáneas a la Formación Palo Pintado. Starck y Anzótegui (2001) sostienen que la Formación Palo Pintado se depositó bajo condiciones climáticas relativamente más húmedas que las del resto de la región y que este aporte de humedad facilitó el desarrollo de lagunas y pantanos (Galli et al., 2010, 2011). A pesar de una variada evidencia de mayor humedad en la Formación Palo Pintado (BywaterReyes et al., 2010; Galli et al., 2011; Bona et al., 2014; Anzótegui et al., 2017; Robledo, 2017; Zimicz et al., 2018), todavía no queda claro el o los factores que determinaron estas condiciones ambientales. No obstante, Starck y Anzótegui (2001) sugieren que los vientos húmedos provenientes del Atlántico se habrían encontrado con barreras orográficas, lo que pudo generar que estos descargaran su humedad y provocaran intensas lluvias en la región.

Por otra parte, tradicionalmente en la paleobotánica se utiliza el afín actual más cercano de una especie fósil como método para inferir los climas pasados. Sin embargo, el inconveniente que puede surgir con este método es que las asignaciones taxonómicas resulten erróneas, por lo tanto, las inferencias paleoclimáticas se alterarían (Wolfe y Hopkins,
1967; Dilcher, 1971, 1973). Debido a lo anterior, en las últimas décadas ha resurgido un método alternativo del cual se pueden obtener condiciones paleoclimáticas independientes de la taxonomía (Roth y Dilcher, 1978; Wing y Greenwood, 1993; Su et al., 2010; Hinojosa et al., 2011; Peppe et al., 2011; Breedlovestrout et al., 2013; Hernández-Villalva et al., 2013; Spicer et al., 2016). Este método consiste en el análisis de la fisionomía foliar, que se centra en el estudio de las características del margen y el tamaño del área foliar. Bailey y Sinnott (1916), en un trabajo pionero, proponen que la mayor proporción de hojas con margen entero se relaciona de forma positiva con la temperatura, es decir, que se hallará un mayor porcentaje de hojas con margen entero en regiones tropicales y fisiológicamente secas que en zonas templadas. De la misma forma, Raunkiaer (1934) demostró que la mayor humedad del suelo se relaciona directamente con la proporción de hojas de mayor área foliar. De este modo, concluyó que existe una correlación positiva entre el tamaño del área de la hoja y las precipitaciones.

El objetivo de este trabajo es analizar la fisonomía de las hojas fósiles procedentes de la Formación Palo Pintado (Mioceno superior), a fin de obtener la temperatura media anual (TMA) y la precipitación media anual (PMA). De esta forma se podría comprobar la hipótesis de que en la región existió un ambiente húmedo a causa de las lluvias que propiciaron la permanencia de los cuerpos de agua. Los resultados preliminares del análisis (alta proporción de hojas de margen entero y sobre todo leptófilas/micrófilas) arrojarían valores elevados de TMA y PMA, coincidentes con el clima inferido previamente a través del registro fósil y sedimentológico.

\subsection{Marco geológico}

La Formación Palo Pintado aflora en la provincia de Salta, entre las localidades de San Carlos y Angastaco, y está constituida por una secuencia de rocas sedimentarias de origen fluvial de $\sim 1.200 \mathrm{~m}$ de espesor (Fig. 1). Yace de manera concordante sobre la Formación Angastaco (Mioceno medio) y su contacto superior es concordante con la Formación San Felipe (Plioceno). La secuencia sedimentaria granodecreciente, formada por conglomerados, areniscas y rocas pelíticas, ha sido interpretada como un sistema fluvial con depósitos desarrollados dentro del canal o intracanal y de 

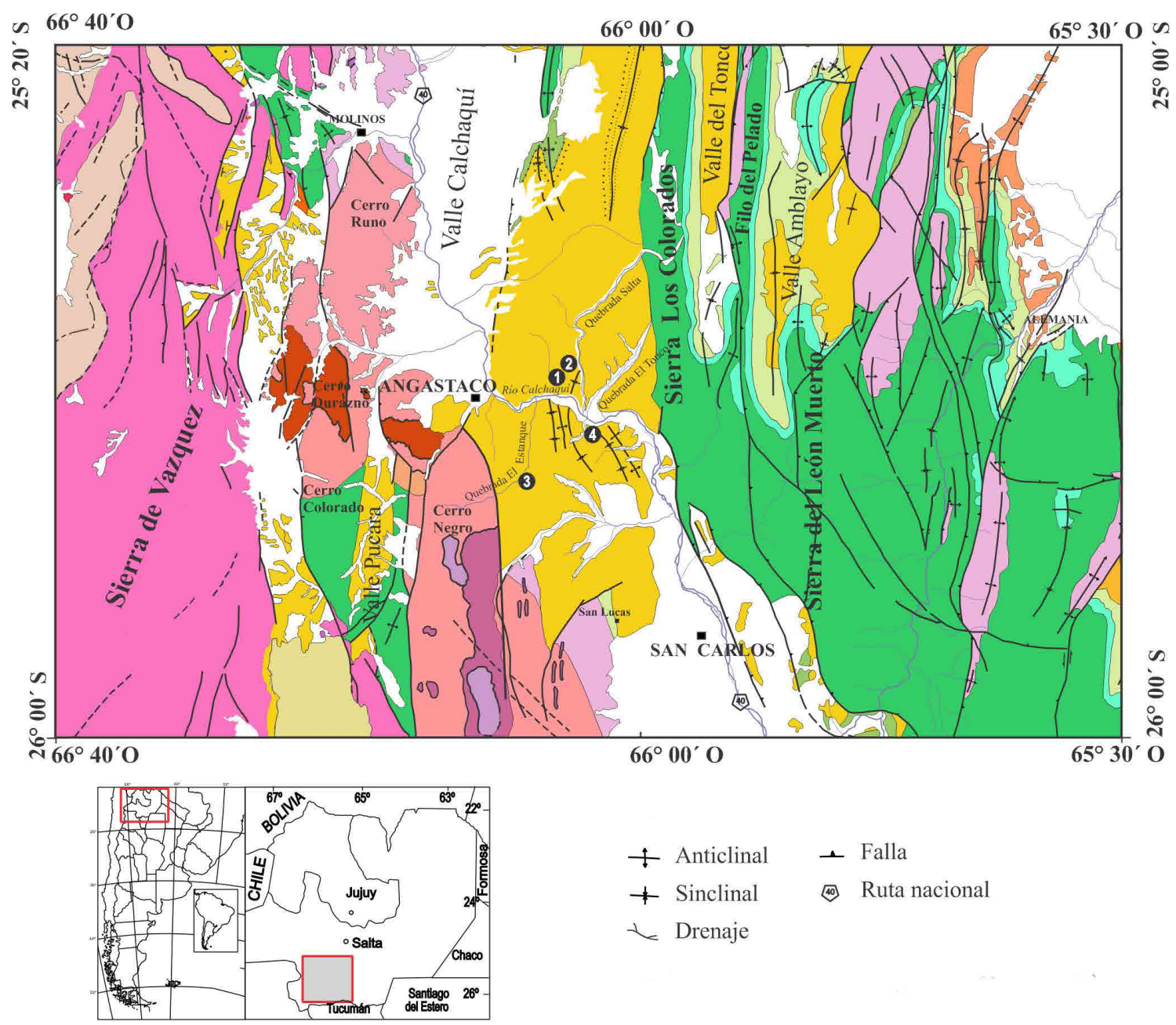

$$
\begin{aligned}
& + \text { Anticlinal }+ \text { Falla } \\
& + \text { Sinclinal } \text { « Ruta nacional } \\
& + \text { Drenaje }
\end{aligned}
$$

$\square$ Formación Puncoviscana (Proterozoico-Cámbrico)
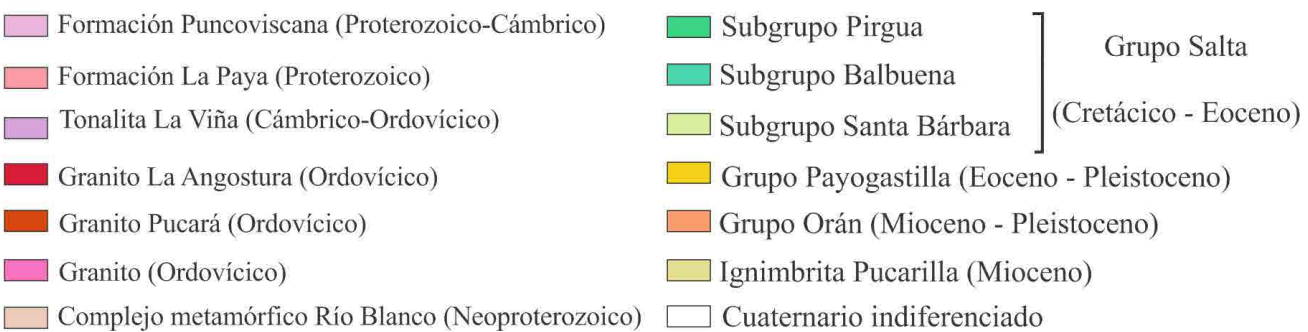

FIG. 1. Mapa geológico que señala la ubicación de las localidades donde provienen las muestras. 1. Peñas Blancas; 2. Quebrada Salta; 3. Quebrada El Estanque; 4. Quebrada del Horno. Modificado de Hong y Seggiaro (2001) y Salfity y Monaldi (2006).

overbank (Galli et al., 2011). Las acumulaciones intracanal están compuestas por depósitos de barras gravosas y formas de lecho que se relacionan con barras transversas y sand wave. Por otro lado, los depósitos de overbank están representados por los siguientes elementos: a) barras de acreción lateral, en donde la superposición lateral y vertical muestra repetidos procesos de erosión y acreción lateral en la llanura de inundación; $\mathbf{b}$ ) canales pequeños, producto de la erosión en los bordes del canal principal durante eventos de inundación, que son asignados a crevasse channel y c) desarrollo de una amplia llanura de 
inundación (Galli et al., 2011). La presencia de minerales de arcilla en el subambiente de llanura de inundación, como illita, esmectita y caolinita indican que han sido generados por hidrólisis en un clima templado-húmedo (Starck y Anzótegui, 2001; Galli et al., 2011, 2014a y b). La formación de estos niveles arcillosos durante la depositación de la Formación Palo Pintado, evidencia un cambio climático importante entre los 10 y $5 \mathrm{Ma}$, que no se registra en los niveles más antiguos de la misma cuenca de depósito (Galli et al., 2017).

Tanto la correlación estratigráfica (Miserendino Fuentes y Díaz, 1988) como las dataciones radiométricas (Grier y Dallmeyer, 1990; Galli et al., 2008; Coutand et al., 2006) confieren a la Formación Palo Pintado una edad del Mioceno superior-Plioceno inferior. Galli et al. (2008) en niveles de tobas datados señalan una edad $10,29 \pm 0,11 \mathrm{Ma}(\mathrm{K}-\mathrm{Ar})$ y para otros próximos al techo se sugiere una edad de 5,27 $\pm 0,28 \mathrm{Ma}(\mathrm{U}-\mathrm{Pb}$ en circón) (Coutand et al., 2006).

\subsection{Antecedentes paleobotánicos y geológicos del área}

Garralla et al. (2016) sostienen que durante el Mioceno-Plioceno se distinguen dos situaciones ambientales en el noroeste argentino que comprenden los afloramientos de las Formaciones Anta, San José, del Buey, Chiquimil, Playa del Zorro, pozo YPF.SE. LH.x-2 Los Horcones, Palo Pintado y Andalhuala. Por un lado, la prevalencia de condiciones secas desde el Mioceno al Plioceno, y por el otro, un mejoramiento de las condiciones climáticas, más húmedas en el momento de la depositación de las rocas pertenecientes a las formaciones San José y Palo Pintado; incluso en el caso de la última, predominan los bosques de ribera y pantanos, con escasos representantes xéricos (Starck y Anzótegui, 2001; Galli et al., 2011; Anzótegui et al., 2017). Además, Anzótegui (2006) y Mautino (2010) sostienen que las provincias fitogeográficas actuales del centro-norte de la Argentina (de las Yungas, Paranaense, Chaqueña, del Monte y del Espinal) y del sur de Brasil (Atlántica) no estuvieron delimitadas durante este lapso, como lo están en la actualidad. Por lo que se considera que estas paleofloras contienen antecesores de las actuales provincias fitogeográficas del norte argentino y sur brasileño con bosques en galería, sabanas arboladas o herbáceas.

Por otra parte, estudios de isótopos estables de hidrógeno, en vidrio volcánico de tobas de la
Formación Palo Pintado proporcionaron datos de precipitaciones y aridización de ella (Pingel et al., 2016). La fracción de vidrio de los niveles de ceniza volcánica intercalados en la secuencia sedimentaria constituye un proxy ambiental que conserva una señal de la composición isotópica de hidrógeno en la precipitación antigua. Esta composición isotópica ayuda a dilucidar los procesos climáticos y tectónicos combinados, los cuales están asociados a la evolución topográfica, que en última instancia controla los patrones espaciales de la precipitación en los cinturones montañosos. Las variaciones de la composición isotópica de hidrógeno, asociadas con el cambio paleoambiental (hace aproximadamente unos $13 \mathrm{Ma}$ ) que significó la formación de la cuenca de Angastaco como una cuenca intermontana limitada por fallas durante el Mioceno-Plioceno tardío (Pingel et al., 2016), sugieren que: a) las condiciones ambientales de la cuenca de Angastaco se vuelven semiáridas, propensas al calentamiento solar y ubicadas en elevaciones transicionales en el antepaís (Strecker et al., 2009; Pingel et al., 2016); y/o b) las condiciones atmosféricas no favorecieron la lluvia convectiva (Pingel et al., 2016). La Formación Palo Pintado junto con la Formación San Felipe (de edad pliocena) están excepcionalmente bien expuestas (Rohrmann et al., 2016) y presentan una historia estructural conocida en detalle (Carrera y Muñoz, 2008; Trimble et al., 2008; Galli et al., 2014a). Según Rohrmann et al. (2016), previo a la depositación de la Formación Palo Pintado, existían condiciones áridas en la zona, las que se tornaron más húmedas hacia el Mioceno tardío. Posteriormente se registra una nueva etapa de aridez durante el Plioceno, hace aproximadamente 3,5 Ma (Coutand et al., 2006). Esto se debió al levantamiento de las sierras León Muerto y Los Colorados (Fig. 1), ubicadas al este de la cuenca de Angastaco (donde aflora la Formación Palo Pintado), las que al alcanzar los $2.000 \mathrm{~m}$ de altura bloquearon el $90 \%$ de la humedad que previamente arribaba desde el este hasta esta cuenca (Bookhagen y Strecker, 2008; Hain et al., 2011; Rohrmann et al., 2016). Esta interpretación es objeto de debate, ya que estudios previos evidencian el levantamiento de estas sierras durante la depositación de la Formación Angastaco, en el Mioceno medio tardío (Galli et al., 2014b). Por otro lado, Bywater-Reyes et al. (2010), también sugieren que las condiciones de aridez se habrían originado antes del alzamiento de la exhumación de dichas sierras. 


\section{Materiales y métodos}

Las impresiones provienen de muestras obtenidas en las localidades de Peñas Blancas, Quebrada Salta, Quebrada El Estanque y Quebrada del Horno, donde aflora la Formación Palo Pintado, en la provincia de Salta (Fig. 1). Algunas de las impresiones que fueron analizadas aquí cuentan con una descripción taxonómica y han sido identificadas a nivel de especies (Anzótegui, 1998; Anzótegui y Cristalli, 2000; Anzótegui y Horn, 2011). En general, tienen un buen estado de preservación y no muestran signos de arrastre hasta el lugar de depositación. Entre las especies que más abundan se hallan frondes estériles y fructificadas de Thelypteris interrupta (Willd.) Iwatsuki 1963 (Thelypteridaceae) y hojas de Schinus herbstii Anzótegui 1998 (Anacardiaceae), Scirpitis sp. Anzótegui 2006 (Cyperaceae), Cedrela fissiliformis Anzótegui y Horn 2011 (Meliaceae), Nectandra saltensis Anzótegui 1998 (Lauraceae) y Ficus tressensii Anzótegui 1998 (Moraceae). Para el análisis fisionómico se utilizaron solo hojas de dicotiledóneas, excluyendo las de monocotiledóneas y helechos. Se analizaron 10 ejemplares de cada especie para determinar el tipo de margen foliar (entero o dentado). En el caso de aquellas especies que no sumaban 10 ejemplares se observaron todas las disponibles. Las hojas de margen entero recibieron una puntuación de 1; las de margen dentado 0 y las que presentaron ambos tipos 0,5 . Con estos datos se obtuvo la temperatura media anual con la fórmula propuesta por Peppe et al. (2011), quienes utilizaron muestras provenientes de 92 localidades actuales distribuidas en todo el mundo.

$$
\mathrm{TMA}=20,4 \times p E+4,6
$$

Donde $p E$ corresponde a la proporción de hojas con margen entero. Además, se estimó el error estándar (ES), pero como este valor resultó menor a $2{ }^{\circ} \mathrm{C}$ se utilizó $\pm 2{ }^{\circ} \mathrm{C}$ de ES, como sugiere Wilf (1997). También se obtuvieron otros resultados de TMA a partir de las ecuaciones propuestas por diferentes autores (Wing y Greenwood, 1993; Hinojosa et al., 2011; Breedlovestrout et al., 2013). Para conseguir el valor de la precipitación media anual (PMA), se obtuvieron los tamaños foliares de cada uno de los morfotipos de acuerdo con las categorías de RaunkiaerWebb (Webb, 1959). Las especies que presentaban tamaños de hojas en una sola categoría se las puntuó con un 1, aquellas que mostraron dos categorías diferentes con 0,5 y las que se ajustaban a más de dos categorías se las puntuó con 0,25 en cada una de las categorías correspondientes. Posteriormente se calculó el MlnA (promedio de los logaritmos naturales de las áreas foliares de las especies) (Wilf et al., 1998), que se define según la siguiente fórmula:

$$
\mathrm{M} \ln \mathrm{A}=\Sigma a_{i} p_{i}
$$

Donde $a_{i}$ corresponde a los siete promedios del logaritmo natural de cada una de las categorías de tamaños del área foliar (leptófila $=2,12$; nanófila $=4,32$; micrófila $=6,51$; notófila $=8,01 ;$ megáfila $=9,11$; macrófila $=10,9$; mesófila $=13,1)$ y $p_{i}$ representa las proporciones de especies en cada categoría. Una vez obtenido el valor de $\mathrm{MlnA}\left(\mathrm{mm}^{2}\right)$, se calculó la PMA (cm) a través de la siguiente fórmula (Wilf et al., 1998):

$$
\mathrm{M} \ln \mathrm{A}=1,39 \times \ln (\mathrm{PMA})+0,786
$$

Las hojas fueron analizadas con una lupa binocular marca Nikon, modelo SMZ-445 y fotografiadas con una cámara montada Nikon 590CU. Posteriormente las fotografías fueron procesadas (mejoramiento de las imágenes y toma de medidas) con los programas digitales Micrometrics y Corel Draw (versión x8). El material fósil se encuentra depositado en las Colecciones Paleontológicas de la Universidad Nacional del Nordeste, Dr. Rafael Herbst, bajo las siglas CTES-PB (Corrientes Paleobotánica), entre los números 6654 y 14150.

\section{Resultados}

Se analizaron 20 morfotipos de hojas (Tabla 1), algunas ya identificadas, las que presentan mayormente márgenes enteros y tamaños de áreas que varían desde leptófilas a notófilas (Fig. 2); se observa mayor proporción de micrófilas $(0,525)$, seguidas de las nanófilas $(0,225)$. La TMA obtenida es de $23,98^{\circ} \mathrm{C}$, con un error estándar (ES) de $1,16^{\circ} \mathrm{C}$. Los valores de TMA conseguidos mediante las diferentes ecuaciones propuestas por otros autores arrojan una temperatura máxima de $30,21^{\circ} \mathrm{C}$ y un promedio de $26,66^{\circ} \mathrm{C}$. El MlnA es de 5,65 $\mathrm{mm}^{2}$, mientras que el valor de la PMA corresponde a 33,08 cm (Tabla 2). Por otro lado, en la tabla 1 se observa que en la lista taxonómica se encuentran identificadas solo 10 especies, mientras que 12 restan aún por identificar, a las que se suman frutos, semillas y hojas de monocotiledóneas. 
TABLA 1. LISTADO DE LOS MORFOTIPOS ANALIZADOS.

\begin{tabular}{|c|c|c|c|c|c|}
\hline \multirow{2}{*}{ Especies } & \multirow{2}{*}{$\begin{array}{l}\text { Margen } \\
\text { foliar }\end{array}$} & \multicolumn{4}{|c|}{ Tamaño foliar } \\
\hline & & Leptófilas & Nanófilas & Micrófilas & Notófilas \\
\hline Aeschynomene sp. Hutch, 1964 & 1 & 1 & 0 & 0 & 0 \\
\hline Caesalpinia cf. stuckerti & & 0 & 1 & 0 & 0 \\
\hline Cedrela fossiliformis & 1 & 0 & 0 & 0 & 1 \\
\hline Ficus tressensii & 1 & 0 & 0 & 1 & 0 \\
\hline Indet. 1 & 1 & 0 & 0 & 0 & 1 \\
\hline Indet. 2 & 1 & 0 & 1 & 0 & 0 \\
\hline Indet. 3 & 1 & 0 & 0 & 1 & 0 \\
\hline Indet. 4 & 1 & 0 & 0 & 1 & 0 \\
\hline Indet. 5 & 1 & 0 & 0 & 1 & 0 \\
\hline Indet. 7 & 1 & 0 & 1 & 0 & 0 \\
\hline Indet. 8 & 1 & 1 & 0 & 0 & 0 \\
\hline Indet. 9 & 1 & 0 & 0 & 1 & 0 \\
\hline Indet. 11 & 1 & 0 & 0 & 1 & 0 \\
\hline Indet. 12 & 1 & 0 & 0 & 1 & 0 \\
\hline Inga sp. Mill, 1754 & 1 & 0 & 0,5 & 0,5 & 0 \\
\hline $\begin{array}{l}\text { Malvaciphyllum quenquiadensis Anzótegui y } \\
\text { Cristalli, } 2000\end{array}$ & 0,5 & 0 & 0 & 1 & 0 \\
\hline Nectandra saltensis & 1 & 0 & 0 & 0,5 & 0,5 \\
\hline Sapium haematospermoides & 1 & 0 & 0 & 1 & 0 \\
\hline Schinus herbstii & 0,5 & 0 & 0,5 & 0,5 & 0 \\
\hline Zornia sp. & 1 & 0,5 & 0,5 & 0 & 0 \\
\hline Suma & 19 & 2,5 & 4,5 & 10,5 & 2,5 \\
\hline$p E-p_{i}$ & 0,95 & 0,125 & 0,225 & 0,525 & 0,125 \\
\hline$a_{i}$ & - & 2,12 & 4,32 & 6,51 & 8,01 \\
\hline$a_{i} p_{i}$ & - & 0,265 & 0,972 & 3,417 & 1,001 \\
\hline
\end{tabular}

En la primera columna se identifica la especie y en la segunda se describe el tipo de margen foliar presentes en los morfotipos (margen entero: 1; margen entero y dentado presentes: 0,5 ). Las columnas restantes corresponden a las diferentes categorías de tamaños del área foliar. $\boldsymbol{p} \boldsymbol{E}$ : proporción de margen entero, este término aplica exclusivamente para la segunda columna; $\boldsymbol{p}_{i}$ : proporción de hojas presentes en cada categoría de tamaño foliar; $\boldsymbol{a}_{\boldsymbol{i}}$ : corresponde a las medias de los logaritmos naturales de las áreas para cada categoría (ver Wilf, et al., 1998).

TABLA 2. ESTIMACIÓN DE LA TEMPERATURA MEDIAANUAL (TMA) Y PRECIPITACIÓN MEDIAANUAL (PMA) OBTENIDAS A PARTIR DEL ANÁLISIS FOLIAR, PARA EL ÁREA DE LA CUENCA DE ANGASTACO.

\begin{tabular}{ccccccccc}
\hline Formación & $\begin{array}{c}\mathbf{N}^{\circ} \text { de morfotipos } \\
\text { analizados }\end{array}$ & TMA $^{\mathbf{1}}$ & TMA $^{\mathbf{2}}$ & TMA $^{\mathbf{3}}$ & $\mathbf{T M A}^{\mathbf{4}}$ & $\mathbf{E S}$ & MLNA & PMA \\
\hline Palo Pintado & 20 & 23,98 & 26,03 & 26,43 & 30,21 & 2,00 & 5,65 & 33,08 \\
\hline
\end{tabular}

TMA $^{1}$ : Peppe et al. (2011); TMA²: Hinojosa et al. (2011), aplicado para paleofloras de Sudamérica; TMA ${ }^{3}$ : Breedlovestrout et al. (2013), ecuación obtenida a partir de la base de datos originales del CLAMP (Climate Leaf Analysis Multivariate Program) (Wolfe, 1993); TMA4: Wing y Greenwood (1993), basada en el análisis de hojas colectadas por Wolfe (1979), en 39 localidades al este de Asia. Los valores de MlnA y PMA se expresan en $\mathrm{mm}^{2}$ y cm, respectivamente. ES: corresponde al error estándar. 

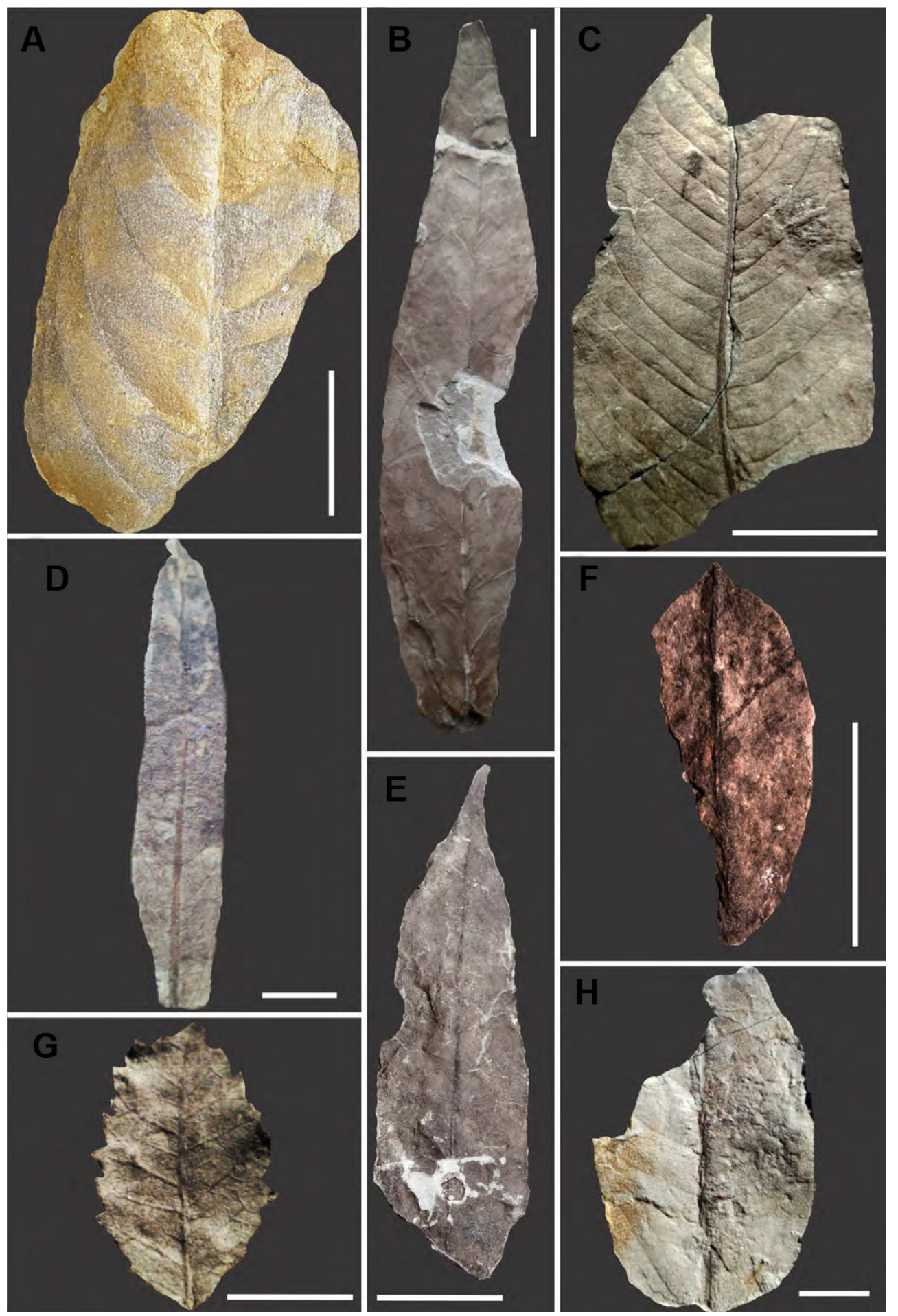

FIG. 2. Impresiones foliares de algunos de los ejemplares estudiados. A. Inga sp. (Fabaceae) (CTES-PB 14129a); B. Nectandra saltensis (Lauraceae) (CTES-PB 14393); C. Dicotiledónea indeterminada (Indet. 4, en la Tabla 1) (CTES-PB 14395); D. Sapium haematospermoides Anzótegui y Horn 2011 (Euphorbiaceae) (CTES-PB 10779b); E. Cedrela fissiliformis (Meliaceae) (CTES-PB 8028a); F. Zornia sp. Gmelin 1791 (Fabaceae) (CTES-PB 14394); G. Schinus herbstii (Anacardiaceae) (CTES-PB 8033); H. Ficus tressensii (Moraceae) (CTES-PB 8076). Escala gráfica: $1 \mathrm{~cm}$. 


\section{Discusión}

Starck y Anzótegui (2001) proponen que durante el periodo de depositación de las rocas sedimentarias que constituyen la Formación Palo Pintado, existía una barrera orográfica al oeste del valle Calchaquí (Fig. 1) que impedía el paso de los vientos húmedos provenientes del Atlántico. En consecuencia, los vientos habrían descargado su humedad posibilitando la existencia de los pantanos formados en las llanuras de inundación durante las estaciones lluviosas (Galli et al., 2011). Starck y Anzótegui (2001) especulan, además, que las lluvias podrían haber alcanzado los $1.000 \mathrm{~mm}$ anuales y que estas condiciones de humedad y temperaturas altas habrían prevalecido aproximadamente hasta los últimos 4 (Starck y Anzótegui) a 3,5 Ma (Coutand et al., 2006), en el límite entre el Plioceno inferior y superior. Durante este periodo de tiempo, tuvo lugar el levantamiento de una nueva barrera orográfica, esta vez al este del valle Calchaquí (configuración que se observa en la actualidad), constituida por las sierras Los Colorados y León Muerto; lo que dejó al valle en una depresión parcialmente aislada (Coutand et al., 2006; Carrapa et al., 2011, 2012; Rohrmann et al., 2016). Bona et al. (2014) sostienen que el levantamiento de este frente orogénico, habría desplazado las condiciones húmedas unos $150 \mathrm{~km}$ hacia el este, lo cual queda confirmado por las similitudes entre la paleoflora encontrada en las rocas sedimentarias de la Formación Palo Pintado (Anzótegui, 1998; Anzótegui y Horn, 2011; Anzótegui et al., 2017) y la flora observada actualmente en el Chaco Occidental (Sarmiento, 1972).

Si se considera que la conformación orográfica actual no presenta cambios desde el Plioceno ( $4 \mathrm{Ma})$, los resultados de este estudio estarían sustentando la existencia de un ambiente de mayor humedad en la cuenca de Angastaco; en comparación a lo que se registra después del levantamiento de las sierras Los Colorados y León Muerto, lo que se ve avalado por los datos actuales de precipitaciones obtenidos en la región de la misma cuenca ( 200 mm anuales) (Galván, 1981; Garleff y Stingl, 1983; Haselton et al., 2002). Si bien estos resultados no indican un ambiente netamente húmedo, se puede concluir que las rocas sedimentarias continentales de la Formación Palo Pintado se habrían depositado bajo condiciones de humedad relativamente altas con precipitaciones que permitieron el desarrollo de cuerpos de agua en las llanuras de inundación de los ríos. Dicha interpretación está sustentada en los registros sedimentológicos y paleobotánicos previamente mencionados. En este último caso, junto con las especies hidrófilas, como por ejemplo Thelypteris interrupta (Thelypteridaceae), Cedrela fissiliformis (Meliaceae), Acrostichum paleoaureum Beauchamp et al., 1973 (Pteridaceae), entre otras, también se registran especies vegetales asociadas a ambientes secos, como Caesalpinia cf. stuckertii Hassl. 1913 (Fabaceae) y los palinomorfos Tubulifloridites antipodica (Asteraceae), Graminidites sp. (Poaceae), Periporopollenites vivianae (Cactaceae), Echiperiporites parviechinatus (Malvaceae), Margocolporites vanwijhei (Fabaceae-Caesalpiniodeae), Equisetosporites sp. (Ephedraceae), Tubulifloridites antipodica (Asteraceae), Chenopodipollis chenopodiaceioides (Chenopodiaceae), Psilatricolporites sp. (Solanaceae), Triorites orbiculatus (Celtidaceae) Striaticolporites gamerroi (Anacardiaceae), Arecipites sp. (Arecaceae) (Horn, 2014), Chenopodiipollis multicavus Anzótegui y Cuadrado (complejo Amaranthaceae/Chenopodiaceae), Retistephanocolpites microreticulatus (Rubiaceae) (Anzótegui y Cuadrado, 1996) y Equisetosporites notensis (Ephedraceae) (Galli et al., 2011). Lo anterior confirma la coexistencia de estas dos paleocomunidades vegetales (hidrófila y xerófila), aunque la comunidad xerófila se habría desarrollado en zonas alejadas de los cuerpos de agua, y la presencia de sus palinomorfos junto a las especies hidrófilas, se debería a la gran capacidad de dispersión que presentan los granos de polen.

En el estudio realizado por Wilf et al. (1998), se analizaron las áreas foliares de ejemplares provenientes de 50 sitios actuales en Estados Unidos, África, América Central y Sudamérica. Una de esas muestras fue colectada en el bosque del Chaco Occidental (Sarmiento, 1972), lo que correspondería a la región del "Chaco Salteño". Si se comparan los resultados obtenidos por Wilf et al. (1998) con los de este trabajo, los valores son similares. La PMA para la región del bosque chaqueño es de 450-700 mm, mientras que en este estudio la PMA indicada por la flora fósil contenida en las rocas de la Formación Palo Pintado es de 330,8 mm. De la misma forma, el MlnA (ln del área foliar) es de $5,85 \mathrm{~mm}^{2}$ para el primer caso y $5,65 \mathrm{~mm}^{2}$ para el segundo. Por último, la TMA actualmente en la región es de $\sim 20{ }^{\circ} \mathrm{C}$ (Bianchi y Yáñez, 1992), mientras que en la que se depositaron las rocas de la Formación Palo Pintado era de $23,98{ }^{\circ} \mathrm{C}$. Estos resultados confirmarían la interpretación de algunos autores 
(Herbst et al., 1987; Acevedo et al., 1997; Starck y Anzótegui, 2001; Anzótegui, 2006; Galli et al., 2011) que consideran que la secuencia sedimentaria incluida en la Formación Palo Pintado se habría depositado bajo un clima semejante al que se manifiesta en la región del Chaco Occidental.

\section{Conclusiones}

El análisis fisionómico foliar realizado en este trabajo, junto con los registros sedimentológicos y paleobotánicos es una prueba más de que las condiciones ambientales durante la depositación de la Formación Palo Pintado fueron más húmedas que en las regiones cercanas, y presentaron un clima similar al observado actualmente en el Chaco Occidental. Aunque en este caso, el clima habría sido semiárido, con mayor temperatura y menos precipitaciones. Sin embargo, estos valores de precipitaciones fueron suficientes para mantener los cuerpos de agua y la flora asociada a ella.

\section{Agradecimientos}

Los autores quieren agradecer al Dr. F. Hinojosa del Laboratorio de Paleoecología, Facultad de Ciencias, Universidad de Chile, por sus valiosos comentarios; a la Dra. E. Pinheiro del Geological Museum, de la Universidad de Wyoming, por su constante colaboración en la obtención de los resultados; al Dr. I. Contreras del Centro de Ecología Aplicada del Litoral (Argentina), por su aporte en la comprensión de los resultados. Este trabajo fue financiado en el marco del proyecto PI 16F008 (Secretaría General de Ciencia y Técnica de la Universidad Nacional del Nordeste) otorgado a la Dra. L.R. Mautino.

\section{Referencias}

Acevedo, T.L.; Mautino, R.L.; Anzótegui, L.M.; Cuadrado, G.A. 1997. Estudio palinológico de la Formación Palo Pintado (Mioceno Superior), Provincia de Salta, Argentina. Parte II: Esporas. Geociencias 2: 112-120.

Anzótegui, L.M. 1998. Hojas de angiospermas de la Formación Palo Pintado, Mioceno Superior, Salta, Argentina. Parte 1: Anacardiaceae, Lauraceae y Moraceae. Ameghiniana 35: 25-32.

Anzótegui, L.M. 2004. Megaflora de la Formación Chiquimil (Mioceno Superior), en los valles de Santa María y Villavil, provincias de Catamarca y Tucumán, Argentina. Ameghiniana 41 (3): 303-314.
Anzótegui, L.M. 2006. Paleofloras del Mioceno en los valles Calchaquíes, noroeste de Argentina. Ph.D. Thesis (Unpublished), Universidad Nacional del Nordeste, Facultad de Ciencias Exactas, Naturales y Agrimensura: 266 p.

Anzótegui, L.M.; Cuadrado, G.A. 1996. Palinología de la Formación Palo Pintado, Mioceno superior, Provincia de Salta, República Argentina. Parte I: Taxones nuevos. Revista Española de Micropaleontología 28: 77-92.

Anzótegui, L.M.; Cristalli, P. 2000. Hojas de Malvaceae en el Neógeno de Argentina y Brasil. Ameghiniana 37: 169-180.

Anzótegui, L.M.; Horn, M.Y. 2011. Megaflora de la Formación Palo Pintado (Mioceno Superior) Salta, Argentina. Parte II. Revista Brasileira de Paleontología 14 (3): 239-254.

Anzótegui, L.M.; Mautino, L.R.; Garralla, S.S.; Herbst, R.; Robledo, J.M.; Horn, M.Y. 2017. Paleovegetación cenozoica del Noroeste Argentino. In Ciencias de la Tierra y Recursos Naturales del NOA. In Relatorio del Congreso Geológico Argentino, No. 20 (Muruaga, C.; Grosse, P.; editores). Asociación Geológica Argentina: 767-781. Tucumán.

Bailey, I.W.; Sinnott, E.W. 1916. The climatic distribution of certain types of angiosperm leaves. American Journal of Botany 3 (1): 24-39.

Bianchi, A.; Yáñez, C. 1992. Las precipitaciones en el Noroeste Argentino. Segunda Edición. Instituto Nacional de Tecnología Agropecuaria, Estación Experimental Agropecuaria: 383 p. Salta.

Bona, P.; Starck, D.; Galli, C.I.; Gasparini, Z.; Reguero, M. 2014. Caiman cf. latirostris (Alligatoridae, caimaninae) in the late Miocene Palo Pintado Formation, Salta province, Argentina, Paleogeographic and Paleoenvironmental consideration. Ameghiniana 51: 26-36.

Bookhagen, B.; Strecker, M.R. 2008. Orographic barriers, high-resolution TRMM rainfall, and relief variations along the eastern Andes. Geophysical Research Letters 35 (6): L06403. doi: 10.1029/2007GL032011.

Breedlovestrout, R.L.; Evraets, B.J.; Parrish, J.T. 2013. New Paleogene paleoclimate analysis of western Washington using physiognomic characteristics from fossil leaves. Palaeogeography, Palaeoclimatology, Palaeoecology 392: 22-40.

Bywater-Reyes, S.; Carrapa, B.; Clementz, M.; Schoenbohm, L. 2010. Effect of late Cenozoic aridification on sedimentation in the Eastern Cordillera of northwest Argentina (Angastaco basin). Geology 38 (3): 235-238.

Carrapa, B.; Trimble, J.; Stockli, D. 2011. Patterns and timing of exhumation and deformation in the 
Eastern Cordillera of NW Argentina revealed by (U-Th)/He thermochronology. Tectonics 30: TC3003. doi: 10.1029/2010TC002707.

Carrapa, B.; Bywater-Reyes, S.; DeCelles, P.G.; Mortimer, E.; Gehrels, G.E. 2012. Late Eocene-Pliocene basin evolution in the Eastern Cordillera of northwestern Argentina $\left(25-26^{\circ} \mathrm{S}\right)$ : Regional implications for Andean orogenic wedge development. Basin Research 24 (3): 249-268.

Carrera, N.; Muñoz, J. 2008. Thrusting evolution in the southern Cordillera Oriental (northern Argentina Andes): Constraints from growth strata. Tectonophysics 459: 107-122. doiorg/10.1016/j.tecto.2007.11.068.

Coutand, I.; Carrapa, B.; Deeken, A.; Schmitt, A.K.; Sobel, E.; Strecker, M. 2006. Propagation of orographic barriers along an active range front: insights from sandstone petrography and detrital apatite fission-track thermochronology in the intramontane Angastaco basin, NW Argentina. Basin Research 18: 1-26. doi: 10.1111/j.1365-2117.2006.00283.x.

Dilcher, D.L. 1971. A revision of the Eocene flora of southeastern North America. Palaeobotanist 20: 7-18.

Dilcher, D. 1973. A paleoclimatic interpretation of the Eocene floras of Southeastern North America. In Symposium on Vegetation and Vegetational History of Northern Latin America (Graham, A.; editor). Elsevier Scientific Publishing Company.: 39-59. Nueva York.

Galli, C.; Ramírez, A.; Barrientos, C.; Reynolds, J.; Viramonte, J.G.; Idleman, B. 2008. Estudio de proveniencia de los depósitos del Grupo Payogastilla (Mioceno Medio-Superior) aflorantes en el río Calchaquí, provincia de Salta, Argentina. In Congreso Geológico Argentino, No. 17, Actas 1: 353-354. Jujuy.

Galli, C.I.; Vides, M.; Flores, P. 2010. Sedimentological and climatic control in the clay minerals distribution in the fluvial deposits of the Palo Pintado Formation (upper Miocene), Salta Province, Argentina. In International Sedimentological Congress "Sedimentology at the Food of the Andes", No. 18, Resúmenes: 376 p. Mendoza.

Galli, C.I.; Anzótegui, L.M.; Horn, M.Y.; Morton, L.S. 2011. Paleoambiente y Paleocomunidades de la Formación Palo Pintado (Mioceno-Plioceno), provincia de Salta, Argentina. Revista Mexicana de Ciencias Geológicas 28: 161-174.

Galli, C.I.; Coira, L.B.; Alonso, R.N.; Reynolds J.; Matteini, M.; Hauser, N. 2014a. Tectonic controls on the evolution of the Andean Cenozoic foreland basin: Evidence from fluvial system variations in the Payogastilla Group, in the Calchaquí, Tonco and Amblayo valleys, NW Argentina. Journal of South
America Earth Sciences 52: 234-259. doi 10.1016/j. jsames.2014.03.003.

Galli, C.I.; Coira, L.B.; Alonso, R.N.; Matteini, M.; Hauser, N. 2014b. El Grupo Payogastilla (Cenozoico) en los valles Calchaquí, Tonco y Amblayo, provincia de Salta, Argentina. Acta Geológica Lilloana 26 (1): 30-52.

Galli, C.I.; Alonso, R.N.; Coira, L.B. 2017. Integrated stratigraphy of the andean foreland basin (northern Argentina). In Seismic and Sequence Stratigraphy and Integrated Stratigraphy-New Insights and Contributions (Aiello, G.; editor). Intech Open Access: 129-156. doi: $10.5772 / 66242$.

Galván, A.F. 1981. Descripción geológica de la Hoja 10e, Cafayate (Provincias de Tucumán, Salta, y Catamarca). Dirección Nacional de Geología y Minería, Boletín 177: $47 \mathrm{p}$.

Garleff, K.; Stingl, H. 1983. Hangformen und Hangformung in der periglazialen Höhenstufe der argentinischen Anden zwischen $27^{\circ}$ und $55^{\circ}$ Südlicher Breite. In Mesoformen des Reliefs im heutigen Periglazialraum (Poser, H.; Schunke, E.; editores). Abhandlungen Mathematisch-Physikalische Klasse der Akademie der Wissenschaften: 425-434. Göttingen.

Garralla, S.S.; Morton, L.S.; Anzótegui, L.M.; Moyano, S.; Herbst, R. 2012. Primeros registros paleontológicos de la localidad de Cerro Pampa (Formación Chiquimil, Mioceno Tardío), Provincia de Catamarca, Argentina. Gaea: Journal of Geoscience 8 (1): 18-25. doi: 10.4013/ gaea.2012.81.03.

Garralla, S.S.; Anzótegui, L.M.; Mautino, L.R. 2016. Relaciones paleoflorísticas del Mioceno-Plioceno del norte argentino. Publicación Electrónica de la Asociación Paleontológica Argentina 16 (1): 1-13.

Grier, M.E.; Dallmeyer, R.D. 1990. Age of the Payogastilla Group: Implications for foreland basin development, NW Argentina. Journal of South American Earth Sciences 3: 269-278.

Hain, M.P.; Strecker, M.R.; Bookhagen, B.; Alonso, R.N.; Pingel, H.; Schmitt, A.K. 2011. Neogene to Quaternary broken foreland formation and sedimentation dynamics in the Andes of NW Argentina ( $\left.25^{\circ} \mathrm{S}\right)$. Tectonics 30 : TC2006.

Haselton, K.; Hilley, G.E.; Strecker, M.R. 2002. Average Pleistocene climatic patterns in the southern central Andes: controls on mountain glaciation and paleoclimate implications. The journal of Geology 110 (2): 211-226. doi: $10.1086 / 338414$.

Herbert, T.D.; Lawrence, K.T.; Tzanova, A.; Peterson, L.C.; Caballero-Gill, R.; Kelly, C.S. 2016. Late Miocene 
global cooling and the rise of modern ecosystems. Nature Geoscience 9 (11): 843-847.

Herbst, R.; Anzótegui, L.M.; Jalfin, G. 1987. Estratigrafía, paleoambientes y dos especies de Salvinia Adanson (Filicopsida) del Mioceno superior de Salta, Argentina. Facena 7: 15-42.

Hernández-Villalva, D.V.; Calvillo-Canadell, L.; CevallosFerriz, S.R. 2013. Inferencia del paleoclima de la Formación miocénica en Ixtapa Chiapas, utilizando la fisionomía foliar de los fósiles. Paleontología Mexicana 63: 48-65.

Hinojosa, L.F.; Pérez, F.; Gaxiola, A.; Sandoval, I. 2011. Historical and phylogenetic constraints on the incidence of entire leaf margins: insights from a new South American model. Global Ecology and Biogeography 20 (3): 380-390.

Hongn, F.D.; Seggiaro, R.E. 2001. Hoja Geológica 2566III, Cachi, 1:250.000. Instituto de Recursos Minerales. Servicio Geológico Minero Argentino, Boletín 248: 96 p. Buenos Aires.

Horn, M.Y. 2014. Palinofloras de las formaciones El Morterito y Palo Pintado (Mioceno Superior-Plioceno Inferior), noroeste de Argentina. Ph.D. Thesis (Unpublished), Universidad Nacional de La Plata, Facultad de Ciencias y Museo: $141 \mathrm{p}$.

Iwatsuki, K.1963. Thelypteriod ferns of Thailand and Laos collected by Dr. T. Tuyama in 1957-58. The Journal of the Japanese Botany 38 (10): 313-315.

Mautino, L.R. 2010. Palinofloras de las formaciones San José y Chiquimil (Mioceno medio y superior), Noroeste de Argentina. Ph.D. Thesis (Unpublished), Universidad Nacional del Nordeste: 444 p.

Miserendino Fuentes, A.; Díaz, J.I. 1988. Nuevas consideraciones sobre la edad y correlaciones del Grupo Payogastilla (Terciario Superior, Prov. de Salta, Rep. Argentina). In Congreso Geológico Chileno, No. 5, Actas 2: 69-86. Santiago.

Peppe, D.J.; Royer, D.L.; Cariglino, B.; Oliver, S.Y.; Newman, S.; Leight, E.; Enikolopov, G.; FernándezBurgos, M.; Herrera, F.; Adams, J.M.; Correa, E.; Currano, E.D.; Erickson, J.M.; Hinojosa, L.F.; Hoganson, J.W.; Iglesias, A.; Jaramillo, C.A.; Johnson, K.R.; Jordan, G.J.; Kraft, N.J.B.; Lovelock, E.C.; Lusk, C.H.; Niinemets, Ü.; Peñuelas, P.; Rapson, G.; Wing, S.L.; Wright, I.J. 2011. Sensitivity of leaf size and shape to climate: global patterns and paleoclimatic applications. New Phytologist 190 (3): 724-739.

Pingel, H.; Mulch, A.; Alonso, R.N.; Cottle, J.M.; Hynek, S.A.; Poletti, J.; Rohrmann, A.; Schmitt, A.K.; Stockli, D.F.; Strecker, M.R. 2016. Surface uplift and convective rainfall along the southern Central Andes (Angastaco Basin, NW Argentina). Earth and Planetary Science Letters 440(C): 33-42. doi: 10.1016/j.eps1.2016.02.009

Raunkiaer, C. 1934. The Life Forms of Plants and Statistical Plant Geography. Oxford University Press: 632 p. Londres.

Robledo, J.M. 2017. Icnología de las asociaciones planta-insecto de paleofloras de las formaciones San José (Mioceno medio), Chiquimil y Palo Pintado (Mioceno tardío) de los valles Calchaquíes. Ph.D. Thesis (Unpublished), Universidad Nacional del Nordeste: 219 p.

Rohrmann, A.; Sachse, D.; Mulch, A.; Pingel, H.; Tofelde, S.; Alonso, R.N.; Strecker, M.R. 2016. Miocene orographic uplift forces rapid hydrological change in the southern central Andes. Scientific reports 6: 35678.

Roth, J.L.; Dilcher, D.L. 1978. Some considerations in leaf size and leaf margin analysis of fossil leaves. Courier Forschungsinst, Senckenberg 30: 165-171.

Salfity, J.A.; Monaldi, C.R. 2006. Hoja Geológica No 2566 IV Metán, 1:250000. Servicio Geológico Minero Argentino, Boletín 319: 74 p. Buenos Aires.

Sarmiento, G. 1972. Ecological and floristic convergences between seasonal plant formations of tropical and subtropical South America. Journal of Ecology 60: 367-410.

Spicer, R.A.; Yang, J.; Herman, A.B.; Kodrul, T.; Maslova, N.; Spicer, T.E.; Aleksandrova, G.; Jin, J. 2016. Asian Eocene monsoons as revealed by leaf architectural signatures. Earth and Planetary Science Letters 449: 61-68.

Starck, D.; Anzótegui, L.M. 2001. The late climatic change persistence of a climatic signal through the orogenic stratigraphic record in northwestern of Argentina. Journal South American Earth Sciences 14: 763-774.

Strecker, M.R.; Alonso, R.N.; Bookhagen, B.; Carrapa, B.; Coutand, I.; Hain, M.; Hilley, G.E.; Mortimer, E.; Schoenbohm, L.; Sobel, E.R. 2009. Does the topographic distribution of the Central Andean Puna Plateau result from climatic or geodynamic processes? Geology 37 (7): 643-646. doi: 10.1130/ G25545A.1.

Su, T.; Xing, Y.W.; Liu, Y.S.; Jacques, F.M.; Chen, W.Y.; Huang, Y.J.; Zhou, Z.K. 2010. Leaf margin analysis: a new equation from humid to mesic forests in China. Palaios 25 (4): 234-238.

Trimble, J.; Carrapa, B.; Stockli, D.; Stutz, J. 2008. New constraints on the timing and magnitude of deformation and basin exhumation in the Eastern Cordillera of NW Argentina. In American Geophysical Union, Fall Meeting, Resúmenes: T53B-1925. San Francisco. 
Webb, L.J. 1959. A physiognomic classification of Australian rain forests. The Journal of Ecology: 551-570.

Wilf, P. 1997. When are leaves good thermometers? A new case for leaf margin analysis. Paleobiology 23 (3): 373-390.

Wilf, P.; Wing, S.L.; Greenwood, D.R.; Greenwood, C.L. 1998. Using fossil leaves as paleoprecipitation indicators: an Eocene example. Geology 26 (3): 203-206.

Wing, S.L.; Greenwood, D.R. 1993. Fossils and fossil climate: the case for equable continental interiors in the Eocene. Philosophical Transactions: Biological Sciences 341 (1297): 243-252.

Wolfe, J.A. 1979. Temperature parameters of humid to mesic forests of eastern Asia and relation to forests of other regions of the Northern Hemisphere and
Australasia. U.S. Geological Survey, Professional Paper 1106: 37 p. Washington.

Wolfe, J.A.; Hopkins, D.M. 1967. Climatic changes recorded by Tertiary land floras in northwestern North America. In Tertiary correlations and climatic changes in the Pacific (Hatai, K., editor): 67-76. Sendai, Sasaki, Japón.

Zachos, J.; Pagani, M.; Sloan, L.; Thomas, E.; Billups, K. 2001. Trenes, Rhythms, and Aberrations in Global Climate 65 Ma. to Present. Science 292: 686-693.

Zimicz, N.; Payrola, P.; Del Papa, C. 2018. New, Late Miocene mammalian assemblage from the Palo Pintado Formation (Northwestern Argentina). Journal of South American Earth Sciences 81: 31-44.

Manuscript received: August 29, 2018; revised/accepted: March 29, 2019; available online: January 31, 2020. 